\section{Commentary: Quality can be economically efficient, but who deserves the credit?}

\author{
Christoph Haller, MD
}

Health care reimbursement needs to account for the quantity, quality, and complexity of care. Many models or a mix of multiple models have been applied worldwide, such as fee for service, capitation, diagnosis-related groups (DRGs), and pay for performance. No approach has been universally considered superior to the others, especially because each model has strengths in different socioeconomic contexts. In this issue of the Journal, Murin and colleagues ${ }^{1}$ report an analysis of early postoperative extubation (fast-track) in patients with congenital heart disease weighing $<7 \mathrm{~kg}$ and its impact on reimbursement in the German DRG system. ${ }^{1}$ Their retrospective analysis shows that their fast-track protocol did improve patient outcomes with regard to postoperative length of stay in the intensive care unit (ICU) and in the hospital, fewer blood transfusions, and a tendency toward lower early mortality in fasttracked patients compared with matched controls who did not undergo early extubation. Total reimbursement was reduced by $27 \%$ in the fast-track group compared with controls. Simultaneously, ICU nursing staff was reduced over the study period while the same case load was maintained. The authors conclude that a DRG-driven reimbursement system does not provide incentives to improve patient care with their fast-track protocol and that it instead penalizes a unit by decreasing total reimbursement.

The study highlights a problem that is not unique to the DRG model: how can reimbursement systems encourage health care practitioners or hospitals to provide high- quality care while not wasting resources? The German DRG system

\footnotetext{
From the Department of Cardiovascular Surgery, Labatt Family Heart Centre, The Hospital for Sick Children, University of Toronto, Toronto, Ontario, Canada.

Disclosures: The author reported no conflicts of interest.

The Journal policy requires editors and reviewers to disclose conflicts of interest and to decline handling or reviewing manuscripts for which they may have a conflict of interest. The editors and reviewers of this article have no conflicts of interest.

Received for publication Nov 11, 2020; revisions received Nov 11, 2020; accepted for publication Nov 13, 2020; available ahead of print Nov 25, 2020.

Address for reprints: Christoph Haller, MD, Division of Cardiovascular Surgery, The Hospital for Sick Children, 555 University Ave, Toronto, Ontario, Canada M5G 1X8 (E-mail: christoph.haller@sickkids.ca).

J Thorac Cardiovasc Surg 2021;162:444-5

$0022-5223 / \$ 36.00$

Copyright (c) 2020 by The American Association for Thoracic Surgery

https://doi.org/10.1016/j.jtcvs.2020.11.047
}

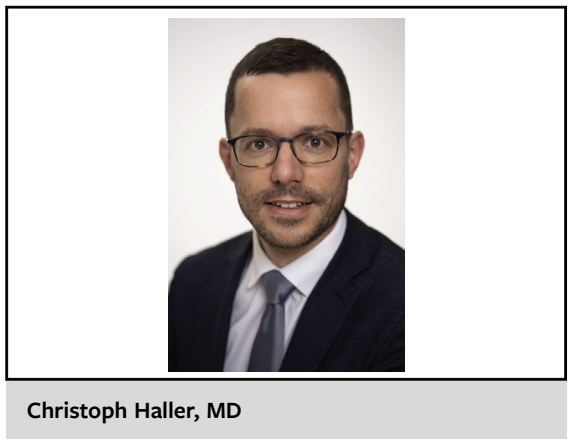

CENTRAL MESSAGE

A system that responds to lower revenue with financial and staffing cuts without taking profit and quality of care into account fails its most fundamental assets: health care professionals.

is based on the International Statistical Classification of Diseases and Related Health Problems-German Modification, which is used to group diagnoses, as well as the operations and procedures key (Operationen und Prozedurenschlüssel) to group procedures. An individual patient's DRG is then determined by multiple factors, such as the main diagnosis, secondary diagnoses, procedures, length of stay, ventilation time, demographic data, and others. Ventilation time is particularly relevant, as certain time thresholds automatically trigger DRGs that significantly increase the case's cost weight (ie, the factor ultimately driving total reimbursement for a particular patient), irrespective of other patient-related factors. Murin and colleagues show that a service that introduces improvements in patient care does not necessarily receive higher reimbursement.

There are several aspects that need to be considered. Most importantly, the study only reflects on reimbursement per se, but does not differentiate between revenue (ie, reimbursement) and profit (ie, reimbursement minus costs incurred). ICU and hospital length of stay were significantly reduced, the transfusion rate was lower, and staffing was reduced with implementation of the fast-track protocol. These factors likely contribute to reduced costs, thereby driving profit. Murin and colleagues point out that case complexity was similar between groups, and so the expected reimbursement should have been the same and increasing the profit margin. However, this perspective is based on the covariates used for propensity matching. The DRG system considers duration of ventilation as a factor 
affecting case complexity and thereby reimbursement. An inclusion criterion of postoperative ventilation time of $\leq 8$ hours rather than inclusion by era before and after implementation of the fast-track protocol further biases results. This obviously must lead to differences in the reimbursement of both groups. Whether ventilation should be considered a complexity increasing factor on its own may be called into question, but the DRG system does not attempt to reimburse via a risk-adjusted bundled payment per diagnosis. It also attempts to adjust for incurred costs, at least to some extent.

Thresholds for ventilation times that trigger higher reimbursement in the German DRG system are set at specific intervals. The risk that the system will incite an intentional delay in extubation or hamper the implementation of fasttrack protocols seems rather low and has been discounted by health insurers. ${ }^{2}$ Surprisingly, a substantial number of patients in the fast-track group were still classified under a DRG associated with ventilation times $>24$ hours. It is also difficult to imagine that the significant differences between groups in the number of patients ventilated for $>95$ hours were driven primarily by the fast-track protocol. A patient who can be safely extubated early postoperatively is likely not ventilated for 4 or more days irrespective of a fast-track protocol.

The authors have shown excellent results in a challenging group of patients and must be congratulated on their success. Despite reduced staffing, they maintained their case load and improved outcomes. What they correctly highlight is a much more complex problem in health care. Economic efficiency and high-quality care can sometimes be conflicting interests. Innovation and progress often do not translate into immediate economic advantage but may turn out to do so in the long term. Especially in congenital cardiac surgery, case volume cannot be increased rapidly, ${ }^{3}$ and regulated reimbursement rates do not allow centres of excellence to charge higher fees. Hospital administration is often cutting budgets and directs resources away from units with lower reimbursement. This is further aggravated by understaffed nursing, a chronic problem driven by decreased labor costs, tough working conditions, and low pay. Collective labor agreements do not differentiate between the complexity of the specialty, with the same salaries for doctors and nursing across specialties. Administration needs to understand that their primary asset is the expertise of their employees and the quality of care they provide. The German health care system undervalues that and needs to provide incentives for health care practitioners at every level to stop the ongoing outflux of qualified professionals. A strict adherence to the Federal Joint Committee guidelines ${ }^{4,5}$ with centralization of care for congenital patients can further increase efficient resource utilization, allow congenital cardiac surgery units to operate economically, and provide the financial scope to reward high-quality care with increased profit margins.

\section{References}

1. Murin P, Weixler VHM, Romanchenko O, Schulz A, Redlin M, Cho MY, et al. Fast-track extubation after cardiac surgery in infants: tug-of-war between performance and reimbursement? J Thorac Cardiovasc Surg. 2021;162:435-43.

2. Biermann A, Geissler A. Beatmungsfälle und Beatmungsdauer in deutschen Krankenhäusern: Eine Analyse von DRG-Anreizen und Entwicklungen in der Beatmungsmedizin. [Incidence and length of ventilation in German hospitals: an analysis of DRG-incentives and developments in respiratory medicine.] Anaesthesist. 2016;65:663-72.

3. Deutsche Herzstiftung, Deutsche Gesellschaft für Kardiologie, Deutsche Gesellschaft für Thorax- Herz und Gefäßchirurgie, Deutsche Gesellschaft für Pediatrische Kardiologie. Deutscher Herzbericht. [German Heart Report.] 2019. Available at: https://www.herzstiftung.de/system/files/2020-11/DHB19_ Herzbericht_2019.pdf. Accessed December 9, 2020.

4. Gemeinsamer Bundesausschuss. Richtlinie des Gemeinsamen Bundesausschusses über Maßnahmen zur Qualitätssicherung der herzchirurgischen Versorgung be Kindern und Jugendlichen gemäß $\S 136$ Abs. 1 Satz 1 Nr. 2 SGB V. [Guideline of the Federal Joint Committee for Provisions for Quality Assurance in Cardiac Surgical Care of Children and Adolescents according to $\$ 136$ Abs. 1 Satz $1 \mathrm{Nr}$. 2 SGB V.]; 2020. 1-24.

5. Deutsche Gesellschaft für Thorax- Herz und Gefäßchirurgie (DGTHG), Deutsche Gesellschaft für Pediatrische Kardiologie (DGPK). Grundvoraussetzungen herzchirurgischer Einheiten zur Behandlung von Patienten mit angeborenen Herzfehlern. [Prerequisites for cardiac surgery units for the treatment of patients with congenital heart defects.] Thorac Cardiovasc Surg. 2016;64:19-24. 